STRUCTURAL BIOLOGY COMMUNICATIONS

ISSN 2053-230X

\section{Expression, purification and crystal structure determination of a ferredoxin reductase from the actinobacterium Thermobifida fusca}

\author{
Jhon Alexander Rodriguez Buitrago, ${ }^{a}$ Thomas Klünemann, ${ }^{b}$ Wulf Blankenfeldt ${ }^{a, b}$ \\ and Anett Schallmey ${ }^{\mathrm{a} *}$
}

Received 9 June 2020

Accepted 6 July 2020

Edited by N. Sträter, University of Leipzig, Germany

Keywords: ferredoxin reductase; Thermobifida fusca; cytochrome P450.

PDB reference: Fdr9, 6tuk

Supporting information: this article has supporting information at journals.iucr.org/f

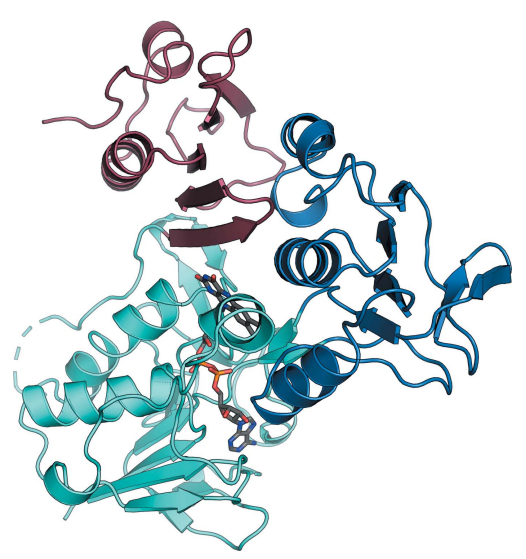
OPEN $\odot$ ACCESS anstitute for Biochemistry, Biotechnology and Bioinformatics, Technical University Braunschweig, Spielmannstrasse 7, 38106 Braunschweig, Germany, and ${ }^{\mathbf{b}}$ Structure and Function of Proteins, Helmholtz Centre for Infection Research, Inhoffenstrasse 7, 38124 Braunschweig, Germany. *Correspondence e-mail: a.schallmey@tu-braunschweig.de

The ferredoxin reductase FdR9 from Thermobifida fusca, a member of the oxygenase-coupled NADH-dependent ferredoxin reductase (FNR) family, catalyses electron transfer from NADH to its physiological electron acceptor ferredoxin. It forms part of a putative three-component cytochrome $\mathrm{P} 450$ monooxygenase system in $T$. fusca comprising CYP222A1 and the [3Fe-4S]cluster ferredoxin Fdx8 as well as FdR9. Here, FdR9 was overexpressed and purified and its crystal structure was determined at $1.9 \AA$ A resolution. The overall structure of FdR9 is similar to those of other members of the FNR family and is composed of an FAD-binding domain, an NAD-binding domain and a C-terminal domain. Activity measurements with FdR9 confirmed a strong preference for NADH as the cofactor. Comparison of the FAD- and NADbinding domains of FdR9 with those of other ferredoxin reductases revealed the presence of conserved sequence motifs in the FAD-binding domain as well as several highly conserved residues involved in FAD and NAD cofactor binding. Moreover, the NAD-binding site of FdR9 contains a modified Rossmann-fold motif, $\mathrm{G} x \mathrm{~S} x x \mathrm{~S}$, instead of the classical $\mathrm{G} x \mathrm{G} x x \mathrm{G}$ motif.

\section{Introduction}

Ferredoxin reductases (FdRs) are essential components of the electron-transfer chains of three-component cytochrome P450 monooxygenases (Hannemann et al., 2007). FdR9 from the actinobacterium Thermobifida fusca belongs to the oxygenasecoupled NADH-dependent ferredoxin reductase family of FAD-dependent electron-transfer enzymes [ferredoxinNAD(P)H reductases; EC 1.18.1.3] (Vorphal et al., 2017). It mediates the transfer of two electrons from NADH to an ironsulfur cluster-containing ferredoxin via two successive oneelectron transfer steps (Medina \& Gómez-Moreno, 2004). Electron transfer between the ferredoxin reductase and the ferredoxin requires the formation of a ternary NADH-FdRFdx complex (Deng et al., 1999; Kuznetsov et al., 2005).

T. fusca is a moderately thermophilic soil bacterium stable enzymes for application in biocatalysis (Wilson, 2004). In the genome of $T$. fusca (Lykidis et al., 2007), the gene coding for FdR9 (Tfu_1273) lies adjacent to the genes encoding CYP222A1 (Tfu_1274) and a putative [3Fe-4S]-cluster ferredoxin named Fdx8 (Tfu_1275). Based on this genomic placement, FdR9, Fdx8 and CYP222A1 are believed to form a three-component cytochrome P450 monooxygenase system. Here, we report the heterologous production, purification, crystallization and structure determination of FdR9, one of (Bachmann \& McCarthy, 1991) and is a rich source of thermo- 
the physiological protein partners of this three-component system. The aim of this study was to provide a structural basis for further investigations of the intermolecular electrontransfer processes between FdR9 and Fdx8 as well as the associated protein-protein interactions.

\section{Materials and methods}

\subsection{Macromolecule production}

The gene encoding FdR9 (Tfu_1273) was provided by Professor Vlada Urlacher (Institute of Biochemistry II, Heinrich-Heine University, Düsseldorf, Germany) in the plasmid pET-22b(+). The gene was subcloned from pET$22 \mathrm{~b}(+)$ into the vector pET-28a(+) using the restriction enzymes NdeI and EcoRI to include an N-terminal $\mathrm{His}_{6}$ tag fused to the resulting protein and a thrombin recognition site between the His tag and the FdR9 sequence. FdR9 was overexpressed in Escherichia coli C43(DE3) cells (Miroux \& Walker, 1996; Dumon-Seignovert et al., 2004) transformed with the Tfu_1273-containing pET-28a(+) vector. For functional expression, the bacteria were grown in $11 \mathrm{~TB}$ medium supplemented with $50 \mathrm{mg}^{-1}$ kanamycin at $37^{\circ} \mathrm{C}$. At an $\mathrm{OD}_{600}$ of 0.8 , expression was induced by the addition of isopropyl $\beta$-D-1-thiogalactopyranoside to a final concentration of $0.5 \mathrm{mM}$. After expression for $16 \mathrm{~h}$ at $240 \mathrm{rev} \mathrm{min}^{-1}$ and $37^{\circ} \mathrm{C}$, the culture was harvested by centrifugation $(15 \mathrm{~min}$ at $4400 \mathrm{~g}$ and $4^{\circ} \mathrm{C}$ ). The cell pellet was resuspended in $24 \mathrm{ml}$ lysis buffer $(20 \mathrm{~m} M$ Tris $\mathrm{pH} 8.0,300 \mathrm{~m} M \mathrm{NaCl})$ in the presence of protease inhibitors (one tablet of Complete EDTA-free per $50 \mathrm{ml}$; Sigma-Aldrich). After sonication (nine cycles of $20 \mathrm{~s}$ at an amplitude of $60 \%$ followed by a $10 \mathrm{~s}$ pause on ice using a Vibra-Cell VCX130, Sonics \& Materials, USA) for cell disruption and subsequent centrifugation (15 min, $10000 \mathrm{~g}$, $4^{\circ} \mathrm{C}$ ) to remove cell debris, FdR9 was found in the soluble fraction. The $\mathrm{His}_{6}$-tag-containing FdR9 was purified by affinity chromatography on an ÄKTAprime FPLC system (GE Healthcare, Freiburg, Germany) using a $5 \mathrm{ml}$ HisTrap column (GE Healthcare). The bound protein was eluted using a linear imidazole gradient $(0-0.5 \mathrm{M})$ in five column volumes $(\mathrm{CV})$. Selected FdR9-containing fractions were combined for incubation with the Thrombin CleanCleave Kit (Sigma-Aldrich) and were then dialyzed against $20 \mathrm{~m} M$ Tris $\mathrm{pH} 8.0,40 \mathrm{~m} M$ $\mathrm{NaCl}$ at $4^{\circ} \mathrm{C}$ for $16 \mathrm{~h}$. Dialyzed samples were again loaded onto a HisTrap column (GE Healthcare) to remove the cleaved His tag. The FdR9-containing flowthrough was loaded onto a $5 \mathrm{ml}$ HiTrap Q HP column (GE Healthcare) and elution was performed using a linear $\mathrm{NaCl}$ gradient $(0.04-$ $1 M)$ in $5 \mathrm{CV}$. Selected FdR9-containing fractions were concentrated by ultrafiltration using a $30 \mathrm{kDa}$ cutoff membrane (Amicon Ultra-15, Merck) and were further purified by gel filtration on a Superdex 75 26/60 column (GE Healthcare) using $20 \mathrm{~m} M$ Tris $\mathrm{pH} 8.0,300 \mathrm{~m} M \mathrm{NaCl}, 1 \mathrm{~m} M$ dithiothreitol. Purified FdR9 was obtained with a yield of $60 \mathrm{mg}$ per litre of culture and displayed a yellow color indicative of the flavin cofactor. A high degree of purity was confirmed for FdR9 by the observation of a single band on a $12 \%$ SDS-PAGE gel.
Table 1

Macromolecule-production information.

\begin{tabular}{|c|c|}
\hline Source organism & T. fusca strain XY \\
\hline Expression vector & pET-28a $(+)$ \\
\hline Expression host & E. coli strain $\mathrm{C} 43(\mathrm{DE} 3)$ \\
\hline \multirow{15}{*}{$\begin{array}{l}\text { Complete amino-acid sequence } \\
\text { of the construct produced }\end{array}$} & MERIVIVGGGLAASRTCEQLRSRGYEGELV \\
\hline & MLCAEPHPPYDRPPLSKAALLEEEHDST \\
\hline & FPTDYAQLSVDVRLGVAATGLVPDARTV \\
\hline & QTTDGELSYDALVIATGASPIRLPGPGR \\
\hline & QFTVRTVEDAAQLRAELKPGQRVVLVGA \\
\hline & SWISAEVATAALRRGCSVTCIEAGPAPL \\
\hline & SAALGADVGQRFLPWWSEVDLRLDTGVA \\
\hline & EVTETGVQLANGEQVDADVVVTGIGVRP \\
\hline & AVDWLSGSGIALDTGVVVDEHLRTSLPG \\
\hline & IYALGDVAVRWSPRWNTRIRVEHWDDAR \\
\hline & EAARTLAGVLLHDPSSQDPLPVHDPVPY \\
\hline & FWSDQFGHKIQYVGHHSPEDTLVIRGDG \\
\hline & TPQWAAAWVDAEGRLTAHLSIDAPRLMI \\
\hline & DARMAIAAGARPDEAALRDPQAKLAPPR \\
\hline & \\
\hline
\end{tabular}

Macromolecule-production information is summarized in Table 1.

\subsection{Crystallization}

Initial crystallization trials were carried out at room temperature in 96-well Intelli-Plates (Art Robbins Instruments, Sunnyvale, California, USA) with the Index screen (D'Arcy et al., 2003) using the sitting-drop vapor-diffusion method. The droplet was initially formed of $200 \mathrm{nl}$ of a solution consisting of $42 \mathrm{mg} \mathrm{ml}^{-1}$ FdR9 in $20 \mathrm{mM}$ Tris $\mathrm{pH} 8.0$, $300 \mathrm{mM} \mathrm{NaCl}$, was mixed with $200 \mathrm{nl}$ reservoir solution using a pipetting robot (Honeybee 963, Genomic Solutions, Huntingdon, UK) and was then equilibrated against $60 \mu \mathrm{l}$ reservoir solution. Crystals of FdR9 were obtained in condition $\mathrm{C} 6$ of the Index screen, consisting of $1.5 \mathrm{M}$ ammonium sulfate, $0.1 M \mathrm{NaCl}, 0.1 M$ bis-Tris $\mathrm{pH}$ 6.5. The crystals were yellow-colored as is typical for native FNR crystals owing to the presence of the flavin cofactor (Morales et al., 2000; Fig. 1). Trials to optimize the crystal quality by varying the precipitant concentration or the $\mathrm{pH}$ did not result in better diffracting crystals. Crystallization information is summarized in Table 2 .

\subsection{Data collection and processing}

FdR9 crystals were harvested using a nylon loop (Hampton Research) and were soaked in reservoir solution containing $20 \%(v / v) 2,3-(R, R)$-butanediol prior to flash-cooling in liquid

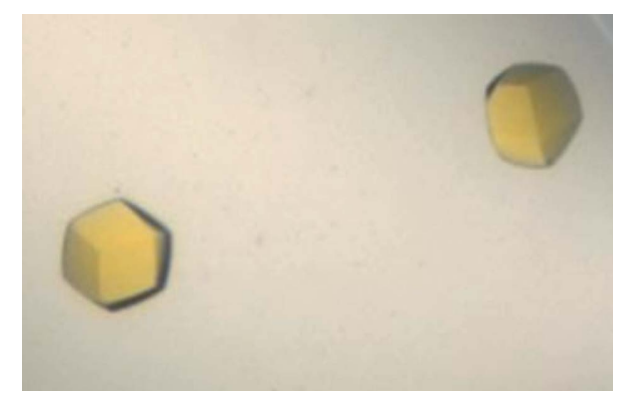

Figure 1

Crystals of ferredoxin reductase FdR9 from T. fusca, with dimensions of $50 \times 50 \times 50 \mu \mathrm{m}$, obtained from Index screen condition C6. 
Table 2

Crystallization.

\begin{tabular}{ll}
\hline Method & Sitting-drop vapor diffusion \\
Plate type & 96 -well Intelli-Plates \\
Temperature $(\mathrm{K})$ & 298 \\
Protein concentration $\left(\mathrm{mg} \mathrm{ml}^{-1}\right)$ & 42 \\
Buffer composition of protein solution & $20 \mathrm{~m} M$ Tris pH $8,300 \mathrm{~m} M \mathrm{NaCl}$ \\
Composition of reservoir solution & $0.1 M \mathrm{NaCl}, 0.1 \mathrm{M}$ bis-Tris $\mathrm{pH} 6.5$, \\
& $1.5 \mathrm{M}$ ammonium sulfate \\
Volume and ratio of drop & $200 \mathrm{nl}, 1: 1$ \\
Volume of reservoir $(\mu \mathrm{l})$ & 60 \\
\hline
\end{tabular}

Table 3

Data collection and processing.

Values in parentheses are for the outer shell.

\begin{tabular}{ll}
\hline Wavelength $(\AA)$ & 1.0332 \\
Temperature $(\mathrm{K})$ & 100 \\
Space group & $P 4_{1} 32$ \\
$a, b, c(\AA)$ & $142.79,142.79,142.79$ \\
$\alpha, \beta, \gamma\left({ }^{\circ}\right)$ & $90,90,90$ \\
Resolution range $(\AA)$ & $142.79-1.90(1.94-1.90)$ \\
Total No. of reflections & $2946416(144375)$ \\
No. of unique reflections & $39768(2527)$ \\
Completeness $(\%)$ & $100(100)$ \\
Multiplicity & $74.1(57.1)$ \\
$\langle I / \sigma(I)\rangle$ & $25.0(2.0)$ \\
CC $1 / 2$ & $1.0(0.704)$ \\
$R_{\text {r.i.m. }}$ & $0.018(0.363)$ \\
Overall $B$ factor from Wilson plot $\left(\AA^{2}\right)$ & 31.74 \\
\hline
\end{tabular}

Table 4

Structure solution and refinement.

Values in parentheses are for the outer shell.

\begin{tabular}{ll}
\hline Resolution range $(\AA)$ & $47.6-1.90(1.97-1.90)$ \\
Completeness $(\%)$ & $99.81(100)$ \\
No. of reflections, working set & $39634(3887)$ \\
No. of reflections, test set & $1951(208)$ \\
Final $R_{\text {cryst }}$ & $0.185(0.243)$ \\
Final $R_{\text {free }}$ & $0.200(0.270)$ \\
No. of non-H atoms & \\
$\quad$ Protein & 2949 \\
$\quad$ Ligand & 91 \\
$\quad$ Solvent & 233 \\
$\quad$ Total & 3273 \\
R.m.s. deviations & \\
$\quad$ Bond lengths $(\AA)$ & 0.010 \\
$\quad$ Angles $\left({ }^{\circ}\right)$ & 0.87 \\
Average $B$ factors $\left(\AA^{2}\right)$ & \\
Protein & 44.15 \\
Ligand & 44.48 \\
$\quad$ Water & 44.99 \\
Ramachandran plot & \\
$\quad$ Most favored $(\%)$ & 97.19 \\
Allowed $(\%)$ & 2.81 \\
$\quad$ Rotamer outliers $(\%)$ & 0.33 \\
\hline
\end{tabular}

nitrogen. 3600 images were collected by the oscillation method with a range of $0.1^{\circ}$ per image on a Dectris PILATUS 6M-F detector using single-wavelength synchrotron radiation on beamline X11 at PETRA III of the Deutsches ElektronenSynchrotron (DESY), Hamburg, Germany. Reflection-image processing was performed using DIALS (Winter et al., 2018) and AIMLESS (Evans \& Murshudov, 2013) from the CCP4 suite (Winn et al., 2011). Data-collection and processing statistics are summarized in Table 3.

\subsection{Structure solution and refinement}

The initial phases were obtained by molecular replacement using MrBUMP (Keegan \& Winn, 2008) executing Phaser (McCoy et al., 2007) and using the atomic coordinates of putidaredoxin reductase (PDB entry 1q1w; Sevrioukova et al., 2004) as a search model. Refinement was performed by alternating rounds of REFMAC5 (Murshudov et al., 2011) and manual adjustments in Coot (Emsley et al., 2010). The final refinement steps were performed with phenix.refine (Afonine et al., 2012), including TLS refinement and the addition of riding $\mathrm{H}$ atoms. The FdR9 diffraction data and coordinates were deposited in the Protein Data Bank (PDB; Berman et al., 2002) as PDB entry 6tuk. Representations of the structures were generated with $P y M O L$ version 2.1.1 (Schrödinger, New York, USA). Refinement statistics are listed in Table 4.

\subsection{Reductase activity assay}

The activity of FdR9 with NADH and NADPH as cofactors was determined spectrophotometrically by measuring the decrease in the ferricyanide concentration at $420 \mathrm{~nm}\left(\varepsilon_{420}=\right.$ $1.02 \mathrm{~m} M^{-1} \mathrm{~cm}^{-1}$; Roome et al., 1983). Each $1 \mathrm{ml}$ reaction contained $0.5 \mathrm{mM} \mathrm{K}_{3} \mathrm{Fe}(\mathrm{CN})_{6}$, an appropriate amount of FdR9 $(3.7 \mu \mathrm{g}$ for the reaction with NADH and $37 \mu \mathrm{g}$ for the reaction with NADPH) and $0.5 \mathrm{~m} M \mathrm{NADH}$ or NADPH in $50 \mathrm{~m} M$ potassium phosphate buffer $\mathrm{pH}$ 7.4. Measurements were performed at ambient temperature using a Cary 60 instrument (Agilent, Heilbronn, Germany).

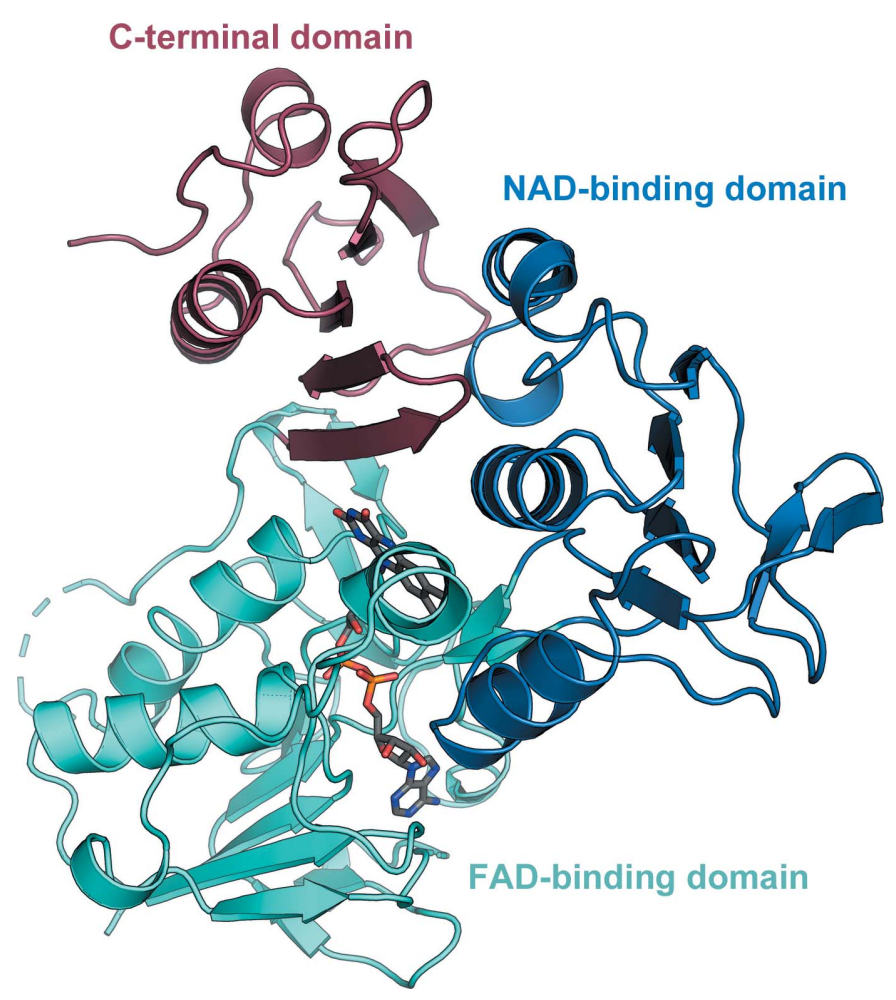

Figure 2

Overall structure of FdR9. The FAD-binding, NAD-binding and C-terminal domains are colored cyan, light blue and light pink, respectively. 


\section{FAD-binding domain}

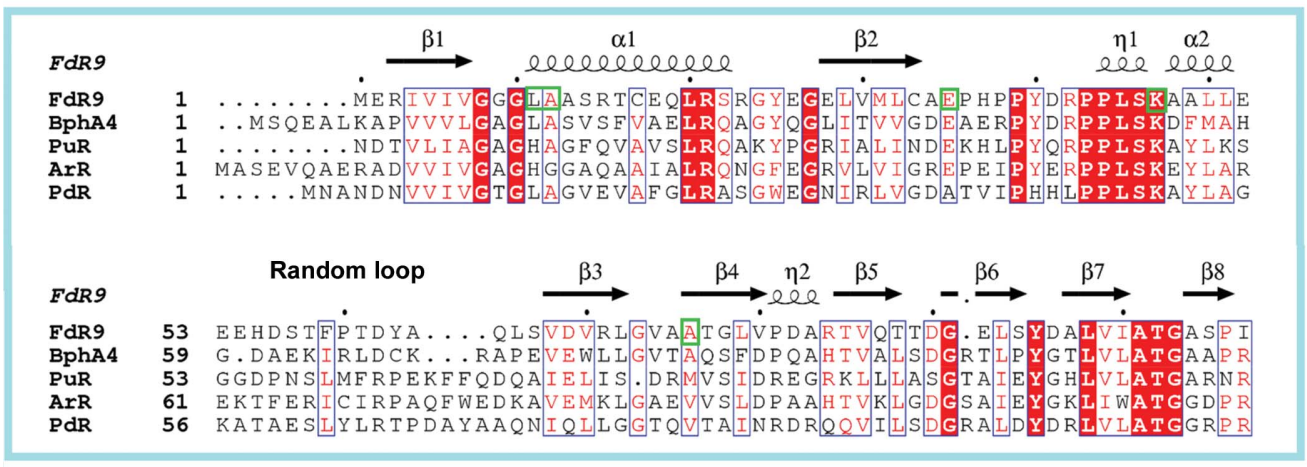

\section{NAD-binding domain}

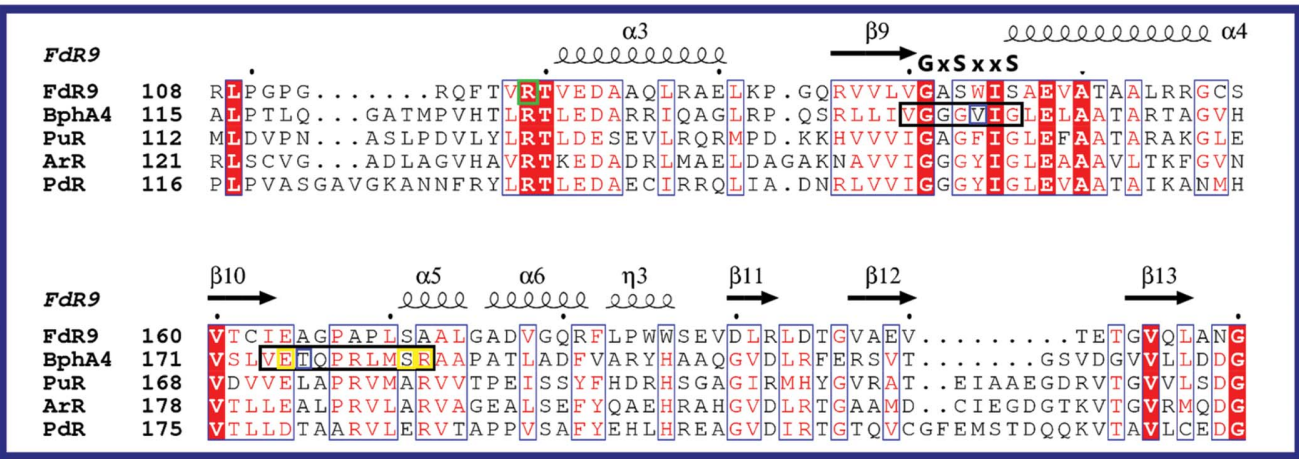
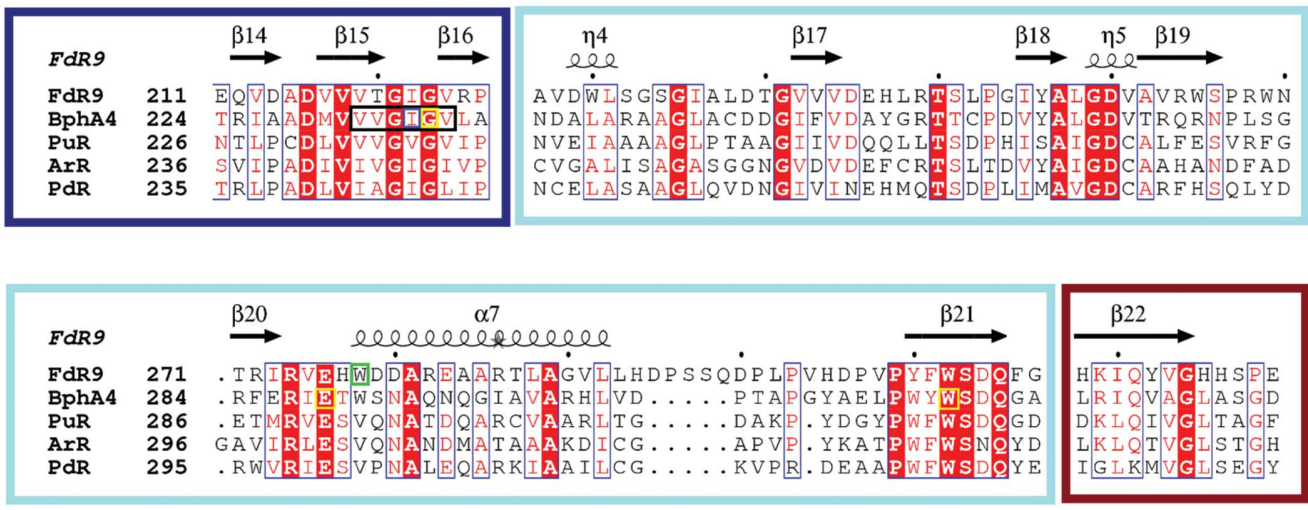

\section{C-terminal domain}

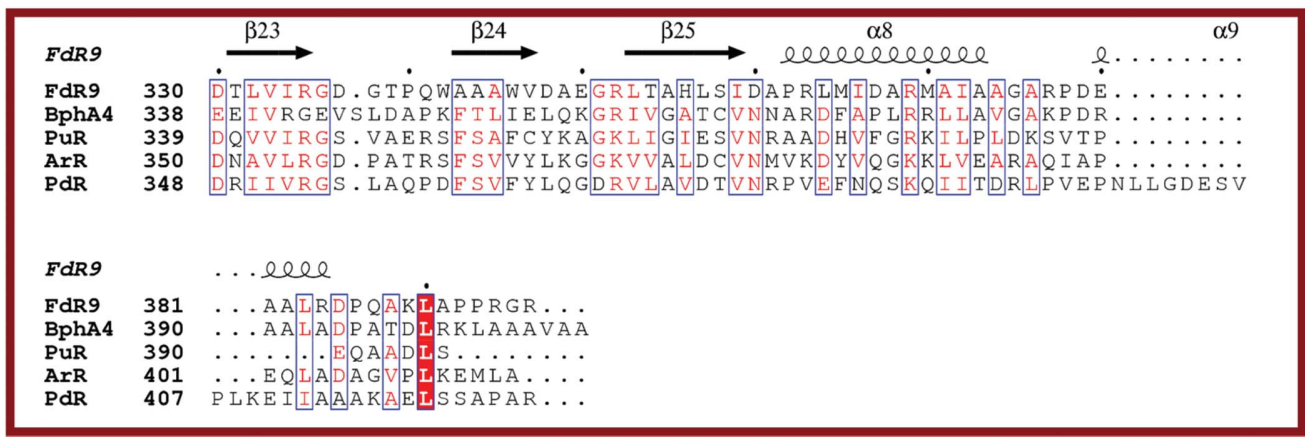

Figure 3

Multiple sequence alignment of FdR9 and homologous ferredoxin reductases obtained from a DALI search using the FdR9 structure as a template. Helices are represented by coils and $\beta$-sheets are shown as arrows. Columns with residues that are more than $70 \%$ similar according to physicochemical properties (threshold set to 0.7 ) are framed in blue and amino-acid residues with $100 \%$ identity are highlighted by a red background. FdR9 residues involved in hydrogen-bond interactions with the FAD molecule are framed in green boxes. The three loops of BphA4 interacting with the NAD molecule are framed in black boxes, and the residues of BphA4 interacting with the NAD molecule by hydrogen bonding and hydrophobic interactions are highlighted by yellow boxes. The PAM250 matrix was used for sequence alignment. The figure was rendered by ESPript 3.0 (Robert \& Gouet, 2014 ). 
Table 5

$D A L I$ search against the PDB using the FdR9 structure.

\begin{tabular}{|c|c|c|c|c|c|c|}
\hline PDB code & Organism & Terminal oxygenase & $\begin{array}{l}\text { Sequence } \\
\text { identity }(\%)\end{array}$ & $\begin{array}{l}\text { Alignment } \\
\text { length }\end{array}$ & $\begin{array}{l}D A L I \\
Z \text {-score }\end{array}$ & Reference \\
\hline $3 f g 2(P u R)$ & Rhodopseudomonas palustris strain CGA009 & CYP199A2 & 27 & 380 & 46.8 & Xu et al. (2009) \\
\hline 3lxd (ArR) & $\begin{array}{l}\text { Novosphingobium aromaticivorans DSM } \\
12444\end{array}$ & $\begin{array}{l}\text { CYP101D1, CYP101B1, CYP101C1, } \\
\text { CYP101D2 }\end{array}$ & 30 & 383 & 47.5 & Yang et al. (2010) \\
\hline 2gqw (BphA4) & Pseudomonas sp. & Biphenyl dioxygenase BphA1A2 & 30 & 382 & 47.2 & Senda et al. (2007) \\
\hline 1q1w (PdR) & Pseudomonas putida & P450cam & 26 & 386 & 46.6 & Sevrioukova et al. (2004) \\
\hline
\end{tabular}

\section{Results and discussion}

\subsection{Structural overview of FdR9}

FdR 9 crystallized in space group $P 4_{1} 32$, with one monomer in the asymmetric unit. The initial phases were obtained by molecular replacement using the structure of putidaredoxin reductase (PDB entry 1q1w; 26\% amino-acid sequence identity) as a search model. The resulting electron-density map allowed the identification of the FAD molecule bound to the reductase. The final model of FdR9, refined to an $R_{\text {cryst }}$ of $18.5 \%$ and an $R_{\text {free }}$ of $20.0 \%$, displays very good geometry, with no residues located in disallowed regions of the Ramachandran plot. The structure of FdR9 contains nine $\alpha$-helices and $25 \beta$-strands, which form three distinct domains (Fig. 2): an FAD-binding domain (residues 1-106 and 223-308), an NAD-binding domain (residues 107-222) and a C-terminal domain (residues 309-393). Structural comparison of FdR9 with other proteins using the DALI server (Holm \& Sander, $1995)$ indicated that it is very similar to ferredoxin reductases from Rhodopseudomonas palustris (PuR), Novosphingobium aromaticivorans (ArR), Pseudomonas sp. (BphA4) and P. putida (PdR) (Table 5), all of which share the three-domain architecture shown in Fig. 2. The root-mean-square deviations (r.m.s.d.s) for $\mathrm{C}^{\alpha}$ atoms of these protein structures from the structure of FdR9 ranged between 1.9 and $2.2 \AA$. PuR, ArR and PdR are part of three-component cytochrome P450 monooxygenase systems, like FdR9, but transfer electrons to [2Fe-2S]-cluster ferredoxins, whereas the physiological interaction partner of FdR9 seems to be a [3Fe-4S]-cluster ferredoxin. In contrast, BphA4 forms part of the three-component biphenyl dioxygenase system present in Pseudomonas sp. (Table 5).

\subsection{FAD-binding domain}

The FAD-binding domain of FdR9 adopts a typical $\alpha / \beta$-fold consisting of two antiparallel $\beta$-sheets and one parallel $\beta$-sheet surrounded by four $\alpha$-helices, as shown in Fig. 2. Sequencealignment analysis of the FAD-binding domain reveals the presence of three highly conserved motifs: PYxRPPLSK, $\mathrm{TS} x \mathrm{P} x_{3} \mathrm{~A} x \mathrm{G}$ and $\mathrm{R} x \mathrm{E} x_{4} \mathrm{~A}$ (Fig. 3). These motifs contain most of the amino acids that interact with the FAD cofactor. In FdR9, the FAD cofactor interacts with the protein through a network of hydrogen bonds to the FAD-binding domain involving amino-acid residues Leu11, Ala12, Glu35, Arg42, Lys47, Ala76, Arg119, Asp260 and Trp278 (Fig. 4), several of which are conserved among the ferredoxin reductases PuR, ArR, PdR and BphA4 (Fig. 3). The pyrophosphate moiety of
FAD is stabilized by hydrogen bonds between the main-chain amide groups of Ala12 and Leu11 and $\mathrm{O}_{1} \mathrm{P}$ and between the side-chain $\mathrm{NH}_{2}$ group of $\mathrm{Arg} 119$ and $\mathrm{O}_{2} \mathrm{~A}$, as well as between the Asp260 side chain and the $\mathrm{O}_{2} \mathrm{P}$ and $\mathrm{O}_{3^{\prime}}$ atoms of the riboflavin part. Moreover, Arg42 plays an important role in stabilizing the FAD cofactor through the formation of electrostatic interactions and hydrogen bonds with the $\mathrm{O}_{1} \mathrm{~A}$ atom of the pyrophosphate moiety and the $\mathrm{O}_{3} \mathrm{~B}$ atom of the adenosine ribose (Fig. 4). In PdR, this arginine is replaced by Leu45, while PuR, ArR and BphA4 also carry an arginine at the respective position (Figs. 3 and 4). Ala76 of FdR9 is involved in stabilization of the adenine through hydrogen bonding of the backbone carbonyl $\mathrm{O}$ atom to the $\mathrm{N}_{6} \mathrm{~A}$ atom. The $\mathrm{O}^{\varepsilon}$ atom of Glu35 forms hydrogen bridges with the $\mathrm{O}_{2} \mathrm{~B}$ and $\mathrm{O}_{3} \mathrm{~B}$ atoms of the adenosine ribose. This glutamate is also conserved in PuR, ArR and BphA4, whereas an alanine is present at the corresponding position in PdR (Figs. 3 and 4). Trp278 stabilizes the isoalloxazine ring of the FAD cofactor by hydrogen bonding between its backbone $\mathrm{NH}$ and the $\mathrm{O}_{2}$ atom of the isoalloxazine ring. Lys47 in FdR9 forms a salt bridge with Glu148 and hydrogen bonds to the carbonyl $\mathrm{O}$ atom of Pro43 as well as to the $\mathrm{O}_{4}$ and $\mathrm{N}_{5}$ atoms of the isoalloxazine ring.

Despite the fact that the overall fold of the FAD domain in FdR9 is highly similar to the structures of PuR, ArR, PdR and

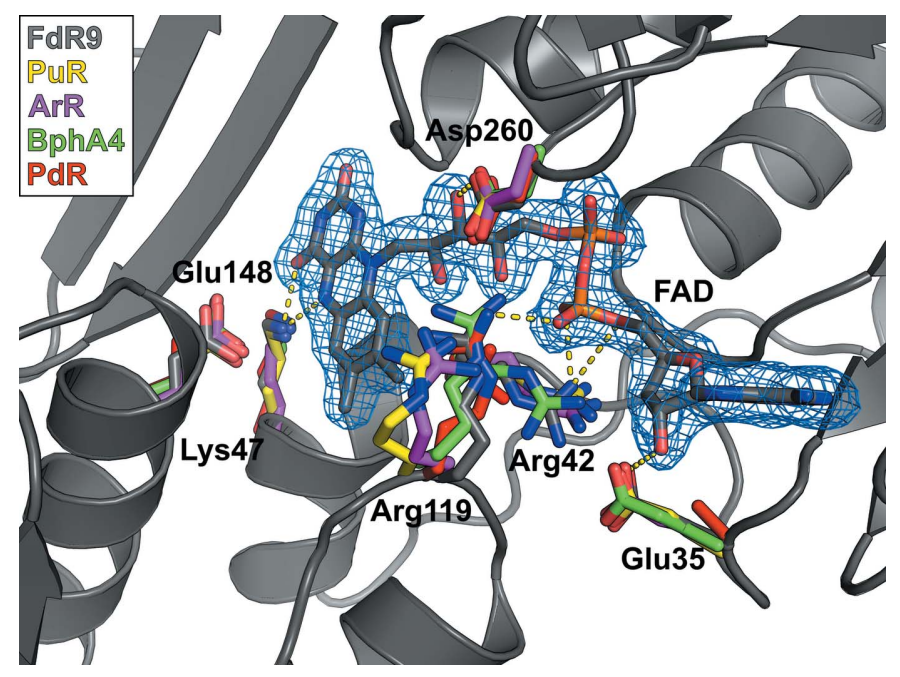

Figure 4

Superposition of the FAD-binding site of FdR9 (PDB entry 6tuk, gray) with the structures of other ferredoxin reductases [PDB entries $3 \mathrm{fg} 2$ (PuR), 3lxd (ArR), 2gqw (BphA4) and 1q1w (PdR)]. Residues forming hydrogen-bond interactions with the FAD cofactor are shown as sticks. Hydrogen bonds are indicated by dotted lines. 
BphA4, with several conserved amino acids interacting with the FAD molecule, differences can also be observed. The first difference involves the presence or absence of secondary structure in the loop region (residues 51-63 in FdR9) of the five ferredoxin reductases. The short and longer helices found in this region in PdR, PuR, ArR and BphA4 correspond to a purely random loop in FdR9 (Fig. 5). It has previously been proposed that the absence of helix secondary structure in this region could influence the binding of FAD (Xu et al., 2009). Another difference is the insertion of five amino acids (residues 295-300) in a surface loop in FdR9, while this loop is

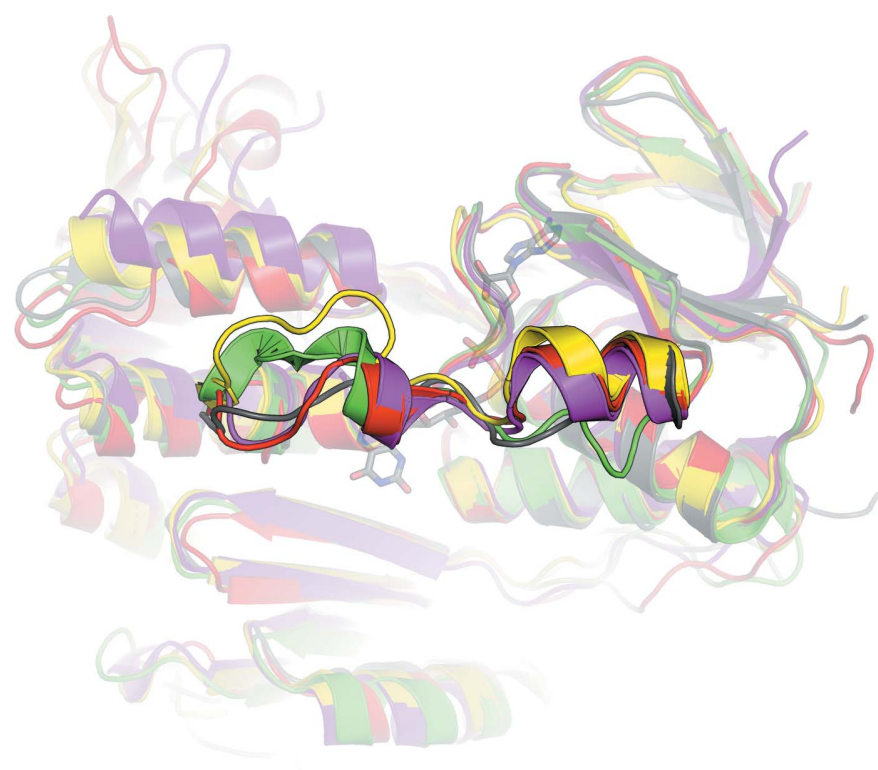

Figure 5

Superposition of the structural model of FdR9 (PDB entry 6tuk, gray) with the structures of other ferredoxin reductases [PDB entries $3 \mathrm{fg} 2$ $(\mathrm{PuR})$ in yellow, 3lxd (ArR) in magenta, 2gqw (BphA4) in green and $1 \mathrm{q} 1 \mathrm{w}(\mathrm{PdR})$ in red] to highlight differences in secondary structure in the random-loop region (residues 51-63 in FdR9). shorter in the other four reductases (Fig. 3). The electron density for residues $298-300$ in this extended loop in FdR9 is not well resolved, indicating high flexibility.

\subsection{NAD-binding domain}

In our FdR9 crystallization experiments we did not attempt to co-crystallize FdR9 with the nicotinamide cofactor, and hence the structure of FdR9 presented here does not contain NAD. Nevertheless, sequence and structural analyses of the NAD-binding domain of FdR9 indicate the presence of a canonical Rossmann fold similar to previously reported complexes, in which the NAD molecule was shown to interact with three loops [corresponding to residues 140-146 (first loop), 164-172 (second loop) and 219-224 (third loop) in FdR9; Fig. 3] (Senda et al., 2000). In PuR, ArR, PdR and BphA4, the first loop between $\beta 9$ and $\alpha 4$ contains the typical $\mathrm{G} x \mathrm{G} x x \mathrm{G}$ motif indicative of nicotinamide cofactor binding, which is modified to GASWIS in FdR9, with the last two glycines in the motif replaced by serines (Fig. 3). This change in the motif does not affect the folding of the respective loop, as can be seen in the structural comparison between FdR9 and BphA4 (Fig. 6). Previous studies proposed that the sequence motif $\mathrm{G} x \mathrm{G} x x \mathrm{G}$ was indicative of NAD specificity, whereas the motif $\mathrm{G} x \mathrm{G} x x \mathrm{~A}$ is found in NADP-binding enzymes, although exceptions are known (Carugo \& Argos, 1997; Hanukoglu, 2017), as would be the case for FdR9. In our studies, FdR9 was found to display a clear preference for NADH as the cofactor. Based on a ferricyanide reduction assay (Roome et al., 1983), the specific activity of FdR9 with NADH was determined to be $104 \mathrm{U} \mathrm{mg}^{-1}$, whereas the specific activity with NADPH was only $1.2 \mathrm{U} \mathrm{mg}^{-1}$. Hence, the nicotinamide cofactor-binding site of FdR9 was compared in more detail with that of BphA4 (PDB entry 1f3p), which was crystallized with NAD bound in the active site. The comparison shows that FdR9 shares a similar fold at the entrance to the NAD-binding channel and an accessible nicotinamide-binding site above the isoallox-

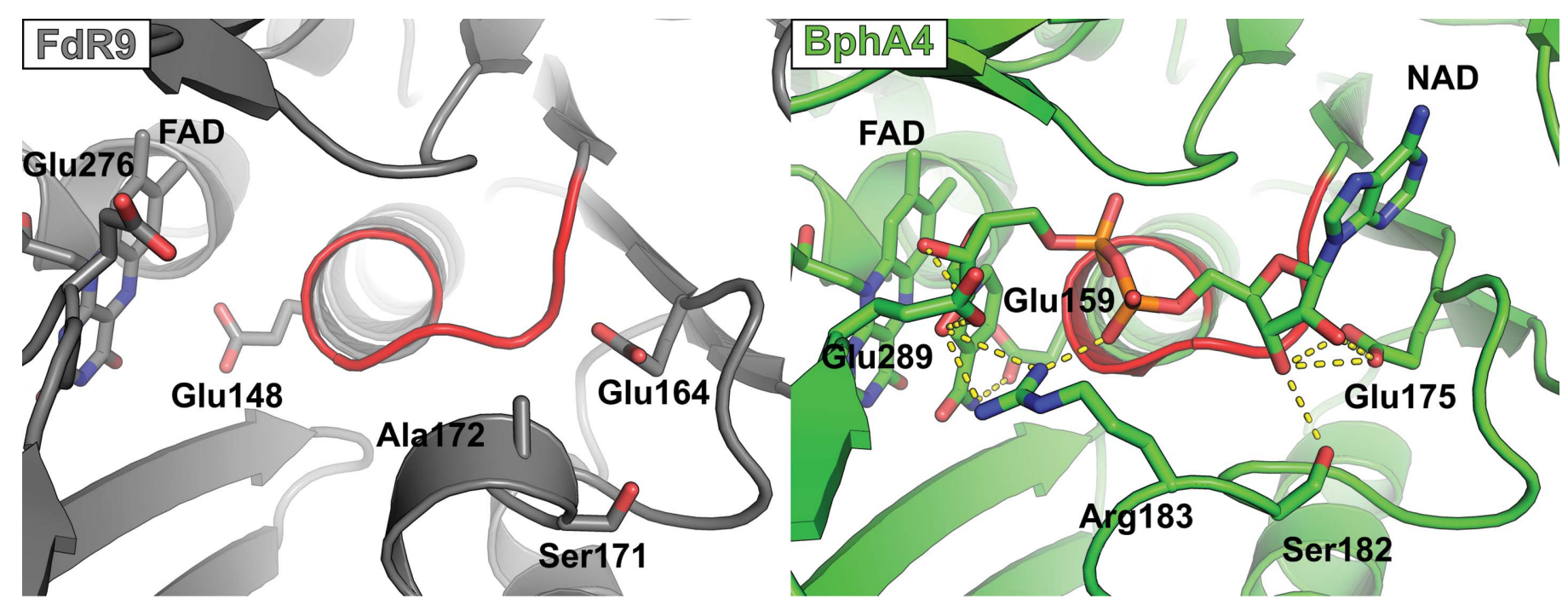

Figure 6

Structural comparison of the NAD-binding sites of FdR9 (PDB entry 6tuk) and the reductase BphA4 (PDB entry 1f3p) in complex with NAD ${ }^{+}$. The amino-acid residues of BphA4 interacting with $\mathrm{NAD}^{+}$and the corresponding residues in FdR9 are labeled. The loops containing the $\mathrm{G} x \mathrm{G} x \mathrm{G}$ motif of BphA4 and the GxS xxS motif of FdR9 are shown in red. 
azine ring of the flavin cofactor. In BphA4, NAD interacts with Val155, Ile156 and Glu159 in the first loop (the corresponding residues in FdR9 are Trp144, Ile145 and Glu148) and with Glu175, Thr176, Ser182 and Arg183 in the second loop (corresponding to Glu164, Ala165, Ser171 and Ala172 in FdR9). Glu175 of BphA4 forms hydrogen bonds to the $\mathrm{O}_{2} \mathrm{~B}$ and $\mathrm{O}_{3} \mathrm{~B}$ atoms of the adenosine ribose as well as to Thr176. The negative charge of Glu175, which is conserved not only in FdR9 (Fig. 6) but also in PuR, ArR and PdR, has been proposed to enhance NAD cofactor specificity by repulsion of the phosphate of NADP (Hanukoglu, 2017). Ser182 in BphA4, which corresponds to Ser171 in FdR9, also forms a hydrogen bond to the adenosine ribose $\mathrm{O}_{3} \mathrm{~B}$ atom. Residues in the third loop interacting with the NAD molecule (Ile235 and Gly236 in BphA4), as well as Glu289 and Trp320 of BphA4, are significantly conserved among the five ferredoxin reductases compared in Fig. 3. Interestingly, FdR9 has a threonine at position 220 (third loop), while PuR, ArR, PdR and BphA4 possess a hydrophobic residue (either valine or alanine) in the corresponding position. Of the 11 amino acids directly interacting with the NAD molecule in BphA4 by hydrogen bonding, eight are conserved in FdR9 (Fig. 3), whereas the proteins share an overall amino-acid sequence identity of only $30 \%$. In contrast, other loop residues (especially in the first and second loop), which do not form direct hydrogen-bond interactions with the nicotinamide cofactor, display a lower degree of conservation.

Apart from this, FdR9 exhibits two significantly shorter surface loops in the NAD-binding domain compared with the other four ferredoxin reductases. This involves the loops between $\beta 8$ and $\alpha 3$ as well as between $\beta 12$ and $\beta 13$ (Fig. 3). The latter in particular is seven, seven and nine amino acids longer in PuR, ArR and PdR, respectively. In PdR this loop is involved in crystallographic dimer formation, while PuR and ArR crystallize as monomers like FdR9 (Sevrioukova et al., 2004; Xu et al., 2009; Yang et al., 2010). It is unclear, however, whether these differences in surface-loop lengths also have a possible physiological impact.

\section{Acknowledgements}

We thank the beamline staff of P11 at the PETRA III synchrotron, Deutsches Elektronensynchrotron (DESY), Hamburg, Germany for granting us access to their facilities. Open access funding enabled and organized by Projekt DEAL.

\section{Funding information}

This project was financially supported by the Deutsche Forschungsgemeinschaft (DFG, German Research Foundation) via the Research Training Group PROCOMPAS (GRK 2223).

\section{References}

Afonine, P. V., Grosse-Kunstleve, R. W., Echols, N., Headd, J. J., Moriarty, N. W., Mustyakimov, M., Terwilliger, T. C., Urzhumtsev, A., Zwart, P. H. \& Adams, P. D. (2012). Acta Cryst. D68, 352-367.
Bachmann, S. \& McCarthy, A. (1991). Appl. Environ. Microbiol. 57, 2121-2130.

Berman, H. M., Battistuz, T., Bhat, T. N., Bluhm, W. F., Bourne, P. E., Burkhardt, K., Feng, Z., Gilliland, G. L., Iype, L., Jain, S., Fagan, P., Marvin, J., Padilla, D., Ravichandran, V., Schneider, B., Thanki, N., Weissig, H., Westbrook, J. D. \& Zardecki, C. (2002). Acta Cryst. D58, 899-907.

Carugo, O. \& Argos, P. (1997). Proteins, 28, 10-28.

D'Arcy, A., Mac Sweeney, A., Stihle, M. \& Haber, A. (2003). Acta Cryst. D59, 396-399.

Deng, Z., Aliverti, A., Zanetti, G., Arakaki, A. K., Ottado, J., Orellano, E. G. \& Karplus, P. A. (1999). Nat. Struct. Biol. 6, 847853.

Dumon-Seignovert, L., Cariot, G. \& Vuillard, L. (2004). Protein Expr. Purif. 37, 203-206.

Emsley, P., Lohkamp, B., Scott, W. G. \& Cowtan, K. (2010). Acta Cryst. D66, 486-501.

Evans, P. R. \& Murshudov, G. N. (2013). Acta Cryst. D69, 1204 1214.

Hannemann, F., Bichet, A., Ewen, K. \& Bernhardt, R. (2007). Biochim. Biophys. Acta, 1770, 330-344.

Hanukoglu, I. (2017). J. Mol. Evol. 85, 205-218.

Holm, L. \& Sander, C. (1995). Trends Biochem. Sci. 20, 478-480.

Keegan, R. M. \& Winn, M. D. (2008). Acta Cryst. D64, 119-124.

Kuznetsov, V. Y., Blair, E., Farmer, P. J., Poulos, T. L., Pifferitti, A. \& Sevrioukova, I. F. (2005). J. Biol. Chem. 280, 16135-16142.

Lykidis, A., Mavromatis, K., Ivanova, N., Anderson, I., Land, M., DiBartolo, G., Martinez, M., Lapidus, A., Lucas, S., Copeland, A., Richardson, P., Wilson, D. \& Kyrpides, N. (2007). J. Bacteriol. 189, 2477-2486.

McCoy, A. J., Grosse-Kunstleve, R. W., Adams, P. D., Winn, M. D., Storoni, L. C. \& Read, R. J. (2007). J. Appl. Cryst. 40, 658-674.

Medina, M. \& Gómez-Moreno, C. (2004). Photosynth. Res. 79, 113 131.

Miroux, B. \& Walker, J. E. (1996). J. Mol. Biol. 260, 289-298.

Morales, R., Kachalova, G., Vellieux, F., Charon, M.-H. \& Frey, M. (2000). Acta Cryst. D56, 1408-1412.

Murshudov, G. N., Skubák, P., Lebedev, A. A., Pannu, N. S., Steiner, R. A., Nicholls, R. A., Winn, M. D., Long, F. \& Vagin, A. A. (2011). Acta Cryst. D67, 355-367.

Robert, X. \& Gouet, P. (2014). Nucleic Acids Res. 42, W320-W324.

Roome, P. W., Philley, J. C. \& Peterson, J. A. (1983). J. Biol. Chem. 258, 2593-2598.

Senda, M., Kishigami, S., Kimura, S., Fukuda, M., Ishida, T. \& Senda, T. (2007). J. Mol. Biol. 373, 382-400.

Senda, T., Yamada, T., Sakurai, N., Kubota, M., Nishizaki, T., Masai, E., Fukuda, M. \& Mitsuidagger, Y. (2000). J. Mol. Biol. 304, 397410.

Sevrioukova, I. F., Li, H. \& Poulos, T. L. (2004). J. Mol. Biol. 336, 889902.

Vorphal, M. A., Bruna, C., Wandersleben, T., Dagnino-Leone, J., Lobos-González, F., Uribe, E., Martínez-Oyanedel, J. \& Bunster, M. (2017). Biol. Res. 50, 39.

Wilson, D. B. (2004). Chem. Rec. 4, 72-82.

Winn, M. D., Ballard, C. C., Cowtan, K. D., Dodson, E. J., Emsley, P., Evans, P. R., Keegan, R. M., Krissinel, E. B., Leslie, A. G. W., McCoy, A., McNicholas, S. J., Murshudov, G. N., Pannu, N. S., Potterton, E. A., Powell, H. R., Read, R. J., Vagin, A. \& Wilson, K. S. (2011). Acta Cryst. D67, 235-242.

Winter, G., Waterman, D. G., Parkhurst, J. M., Brewster, A. S., Gildea, R. J., Gerstel, M., Fuentes-Montero, L., Vollmar, M., MichelsClark, T., Young, I. D., Sauter, N. K. \& Evans, G. (2018). Acta Cryst. D74, 85-97.

Xu, F., Bell, S. G., Peng, Y., Johnson, E. O., Bartlam, M., Rao, Z. \& Wong, L.-L. (2009). Proteins, 77, 867-880.

Yang, W., Bell, S. G., Wang, H., Zhou, W., Hoskins, N., Dale, A., Bartlam, M., Wong, L.-L. \& Rao, Z. (2010). J. Biol. Chem. 285, 27372-27384. 